\title{
Nonparaxial dark solitons in optical Kerr media
}

\author{
Alessandro Ciattoni \\ Istituto Nazionale per la Fisica della Materia, Unità di Ricerca Universitá dell'Aquila, 67010 L'Aquila, Italy, and \\ Dipartimento di Fisica, Universitá dell'Aquila, 67010 L'Aquila, Italy
}

\section{Bruno Crosignani}

Department of Applied Physics, California Institute of Technology, 128-95, Pasadena, California 91125, Universitá dell'Aquila, 67010 L'Aquila, Italy, and Istituto Nazionale per la Fisica della Materia, Unità di Ricerca Roma La Sapienza, o0185 Roma, Italy

\section{Shayan Mookherjea}

Department of Electrical Engineering, Mail Code 0407, University of California, San Diego, La Jolla, California 92093-0407

\section{Amnon Yariv}

Department of Applied Physics, California Institute of Technology, 128-95, Pasadena, California 91125

Received May 18, 2004

We show that the nonlinear equation that describes nonparaxial Kerr propagation, together with the already reported bright-soliton solutions, admits of $(1+1) D$ dark-soliton solutions. Unlike their paraxial counterparts, dark solitons can be excited only if their asymptotic normalized intensity $u_{\infty}^{2}$ is below $3 / 7$; their width becomes constant when $u_{\infty}^{2}$ approaches this value. (C) 2005 Optical Society of America

OCIS codes: $190.0190,190.3270,190.5530$.

Optical spatial solitons are beams in which linear diffraction is exactly compensated for by nonlinearity through self-lensing. This phenomenon has allowed the observation of self-trapping owing to the optical Kerr effect in glass, in polymers, in gases, and in liquids, and also was observed in photorefractive materials and in crystals that exhibit a quadratic (second-order) response., ${ }^{1,2}$ The distinguishing features of solitons are that they allow for guided propagation in an otherwise homogeneous medium, that they manifest quasi-elastic collisions, and that they are amenable to external control through the modulation of launch-wave characteristics, such as intensity or transverse phase chirp. These characteristics, which distinguish them from linear optical propagation, hold the key to potential technological applications, which range from all-optical routing, to transparent beam interconnects, to the massive integration of optical operations in a fully threedimensional environment. In these projected applications, light is made to propagate in effective waveguides that have modes with numerical apertures that violate the paraxial approximation, for which the conventional scalar approach to propagation, such as that at the basis of the parabolic equation, fails. In other words, without drastically reducing the propagating optical wavelength (a solution that encounters a number of hurdles, among which is absorption), miniaturization implies nonparaxial propagation regimes. If solitons are the nonlinear embodiment of optical waveguides, what is the equivalent for submicrometer propagation modes? One possibility that we have been investigating is the direct reduction of the spatial scale for beams propagating in Kerr materials: Can we predict nonparaxial spatial solitons, that is, beams for which diffraction (in the more involved nonparaxial understanding) is compensated for by the similarly more involved Kerr self-action? As the understand- ing of Kerr solitons in a paraxial scheme has triggered a large and fruitful investigation into a wealth of different nonlinear phenomena and processes, so our goal is to set the basis for a similar development in the nonparaxial regime.

The existence of nonparaxial $(1+1) D$ bright spatial solitons in Kerr media, that is, of soliton solutions of the nonlinear Schrödinger equation generalized to include higher-order terms that account for nonparaxial effects, was recently predicted. ${ }^{3}$ These solitons, besides being reduced to standard paraxial solitons in the limit of small normalized peak intensity $u_{0}^{2}$, as expected, present new and interesting features. In particular, the soliton width turns out to be practically independent of the peak intensity for $u_{0}^{2}>1$. It is natural to extend the same analysis to the investigation of nonparaxial dark spatial solitons, which, as is well known, exist in the paraxial regime for defocusing media that possess negative nonlinear refractive-index coefficients $\left(n_{2}<0\right)$. In this Letter we show, by a straightforward generalization of the formalism used for bright solitons, that, whenever $n_{2}<0$, nonparaxial dark solitons exist. However, they differ significantly from their paraxial counterparts in that they can exist only below a specific value of asymptotic intensity $u_{\infty}^{2}\left(u_{\infty}^{2}<3 / 7\right)$.

The governing equation that describes nonlinear propagation in Kerr media of a linearly polarized monochromatic field $E_{x}(x, z, t)=A(x, z) \times$ $\exp (i k z-i \omega t)$ (where $k$ is the wave number in the linear background medium) reads $\mathrm{as}^{4,5}$

$$
\begin{array}{r}
\left(i \frac{\partial}{\partial z}+\frac{1}{2 k} \frac{\partial^{2}}{\partial x^{2}}\right) A=-k \frac{n_{2}}{n_{0}}\left(|A|^{2} A+\frac{1}{3 k^{2}}|A|^{2} \frac{\partial^{2} A}{\partial x^{2}}\right. \\
\left.+\frac{8}{3 k^{2}} A\left|\frac{\partial A}{\partial x}\right|^{2}+\frac{5}{6 k^{2}} A^{2} \frac{\partial^{2} A^{*}}{\partial x^{2}}\right),
\end{array}
$$


which differs from the standard nonlinear Schrödinger equation through the presence on its right-hand side of differential terms that account for the tensorial nature of the nonlinear refractive index, for the nonparaxial effects associated with the transverse scale of variation of the field (comparable with the wavelength), and for the vectorial coupling between transverse and longitudinal components. We introduce the normalized variables $\xi=k x, \zeta=k z, U=\sqrt{\left|n_{2}\right| / n_{0}} A$, to obtain

$$
\begin{aligned}
\left(i \frac{\partial}{\partial \zeta}+\frac{1}{2} \frac{\partial^{2}}{\partial \xi^{2}}\right) U & =-\gamma\left(|U|^{2} U+\frac{1}{3}|U|^{2} \frac{\partial^{2} U}{\partial \xi^{2}}\right. \\
& \left.+\frac{8}{3} U\left|\frac{\partial U}{\partial \xi}\right|^{2}+\frac{5}{6} U^{2} \frac{\partial^{2} U^{*}}{\partial \xi^{2}}\right),
\end{aligned}
$$

where $\gamma=\left|n_{2}\right| / n_{2}=\operatorname{sign}\left(n_{2}\right)$, and look for a soliton solution of the form $U(\xi, \zeta)=\exp (i \beta \zeta) u(\xi)$, thus getting

$$
-\beta u+\frac{1}{2} u^{\prime \prime}=-\gamma\left(u^{3}+\frac{7}{6} u^{2} u^{\prime \prime}+\frac{8}{3} u u^{\prime 2}\right),
$$

where a prime stands for a derivative with respect to $\xi$.

We note that different versions of Eq. (3) exist in the literature ${ }^{6-11}$; the difference from Eq. (3) lies in the coefficients that appear on the right-hand side. The analytical approach presented below and in Ref. 3 would allow, if they were applied to those equations, one to prove the existence of spatial nonparaxial solitons of the same form as our solitons but numerically different in amplitude, width, and nonlinear phase.

The change of dependent variable $f=u^{\prime 2}$ (according to which $\mathrm{d} f / \mathrm{d} \xi=2 u^{\prime} u^{\prime \prime}=u^{\prime} \mathrm{d} f / \mathrm{d} u$ ) reduces Eq. (3) to

$$
\frac{\mathrm{d} f}{\mathrm{~d} u}+\gamma \frac{32 u}{3+7 \gamma u^{2}} f=12 \frac{\beta-\gamma u^{2}}{3+7 \gamma u^{2}} u,
$$

which can be integrated to give, after some algebra,

$$
\begin{aligned}
\left(\frac{\mathrm{d} u}{\mathrm{~d} \xi}\right)^{2}= & \frac{3 \gamma}{8}\left(\beta+\frac{3}{23}\right)-\frac{6}{23} u^{2}+\left[f\left(u_{0}\right)-\frac{3 \gamma}{8}\right. \\
& \left.\times\left(\beta+\frac{3}{23}\right)+\frac{6}{23} u_{0}^{2}\right]\left(\frac{3+7 \gamma u_{0}^{2}}{3+7 \gamma u^{2}}\right)^{16 / 17},
\end{aligned}
$$

where $u_{0}$ is the field amplitude at a given point. This is in itself a remarkable result, as it shows that the problem is, as in the paraxial case, fully integrable. The bright-soliton case was discussed in Ref. 3, where the existence of bright solitons has been proved for $\gamma>$ 0 (no bright soliton exists for $\gamma<0$ ). We consider now the problem of proving the existence of dark solitons. To this end, we write, besides Eq. (5), its derivative with respect to $\xi$, that is,

$$
\begin{aligned}
\frac{\mathrm{d}^{2} u}{\mathrm{~d} \xi}= & -\frac{6}{23} u-16 \gamma\left[f\left(u_{0}\right)-\frac{3 \gamma}{8}\left(\beta+\frac{3}{23}\right)+\frac{6}{23} u_{0}^{2}\right] \\
& \times\left(3+7 \gamma u_{0}^{2}\right)^{16 / 7}\left(3+7 \gamma u^{2}\right)^{-23 / 7} u
\end{aligned}
$$

Taking the limit $\xi \rightarrow \pm \infty$ of both Eqs. (5) and (6), and setting $u_{0}=0, f[u( \pm \infty)]=0$, and $\left(\mathrm{d}^{2} u / \mathrm{d} \xi^{2}\right)_{\xi= \pm \infty}=0$, we get

$$
\begin{aligned}
0= & \frac{3 \gamma}{8}\left(\beta+\frac{3}{23}\right)-\frac{6}{23} u_{\infty}^{2}+\left[f(0)-\frac{3 \gamma}{8}\right. \\
& \left.\times\left(\beta+\frac{3}{23}\right)\right]\left(\frac{3}{3+7 \gamma u_{\infty}^{2}}\right)^{16 / 7}, \\
0= & -\frac{6}{23}-16 \gamma\left[f(0)-\frac{3 \gamma}{8}\right. \\
& \left.\times\left(\beta+\frac{3}{23}\right)\right] \frac{1}{3+7 \gamma u_{\infty}^{2}}\left(\frac{3}{3+7 \gamma u_{\infty}^{2}}\right)^{16 / 7},
\end{aligned}
$$

where $u_{\infty}^{2}=u^{2}( \pm \infty)$. The solution of the set of Eqs. (7) in the two unknowns $\beta$ and $f(0)$ reads as $\beta=\gamma u_{\infty}^{2}$ and

$$
f(0)=\frac{3}{8}\left(u_{\infty}^{2}+\frac{3 \gamma}{23}\right)-\frac{9}{184}\left(1+\frac{7 \gamma}{3} u_{\infty}^{2}\right)^{23 / 7},
$$

which is inserted into Eq. (5), yield

$$
\begin{aligned}
f(u)= & \frac{3}{8}\left(u_{\infty}^{2}+\frac{3 \gamma}{23}\right)-\frac{6}{23} u^{2} \\
& -\frac{3 \gamma}{184}\left(3+7 \gamma u_{\infty}^{2}\right)\left(\frac{3+7 \gamma u_{\infty}^{2}}{3+7 \gamma u^{2}}\right)^{16 / 7} .
\end{aligned}
$$

It is easy to check whether the right-hand side of Eq. (8), is real, as it should be, only if $u_{\infty}^{2}>-3 / 7$ for $\gamma=+1$ and $u_{\infty}^{2}<3 / 7$ for $\gamma=-1$. However, in the first case, $f(u)$, as provided by Eq. (8), is always negative, so there are no dark solitons for $\gamma=+1$ (as in the paraxial limit). In the second case Eq. (8) furnishes

$$
\begin{aligned}
f(u)= & \frac{3}{8}\left(u_{\infty}^{2}-\frac{3}{23}\right)-\frac{6}{23} u^{2} \\
& +\frac{3}{184}\left(3-7 u_{\infty}^{2}\right)\left(\frac{3-7 u_{\infty}^{2}}{3-7 u^{2}}\right)^{16 / 7},
\end{aligned}
$$

which is always positive, so dark solitons exist for $\gamma=-1$ in the range $0<u_{\infty}^{2}<3 / 7$. The results of numerical integration of Eq. (9) are shown in Fig. 1, where $u$ is plotted as a function of $\xi$ for various values of $u_{\infty}$. We can now compare our result with the paraxial result, in which $U(\xi, \zeta)=$ $u_{\infty} \exp \left(i \zeta / \Delta^{2}\right) \tanh (\xi / \Delta)$, with $\Delta^{2}=1 / u_{\infty}^{2}$. In our case the propagation constant is the same, and the relation between $\Delta$ and $u_{\infty}$ (existence curve) turns out to coincide with the paraxial relation only for small values of $u_{\infty}$, as shown in Fig. 2. In particular, for $u_{\infty} \rightarrow 3 / 7, \Delta \rightarrow \sqrt{23 / 6}$ : From an intuitive point of view, the existence of this threshold is related to the eventual dominance of the defocusing effect that is due to nonlinearity over diffraction (which, in our case, has a focusing effect).

Referring, for example, to sodium vapor, which is known to be a strong nonlinear material, one has ${ }^{12} n_{2}=$ $-4 \times 10^{-10} \mathrm{~cm} / \mathrm{V}^{2}$, to which corresponds an asymptotic intensity $I_{\infty}=\left(n_{0} / n_{2}\right)\left(1 / 2 Z_{0}\right) u_{\infty}^{2}$ (where $Z_{0}$ is the vacuum impedance) of $\sim 1 \mathrm{MW} / \mathrm{cm}^{2}$. We have made an extensive numerical investigation of the stability of nonparaxial dark solitons, of which a typical example is shown in Fig. 3. These results provide clear evidence of the robustness and observability of dark solitons, even if they are not a mathematical demonstration of stability, which is beyond the aim of the present study. 


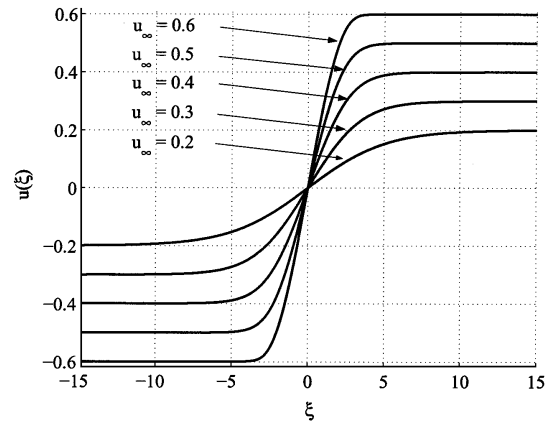

Fig. 1. Soliton envelope $u(\xi)$ and intensity $u^{2}(\xi)$ for various values of $u_{\infty}^{2}<3 / 7$.

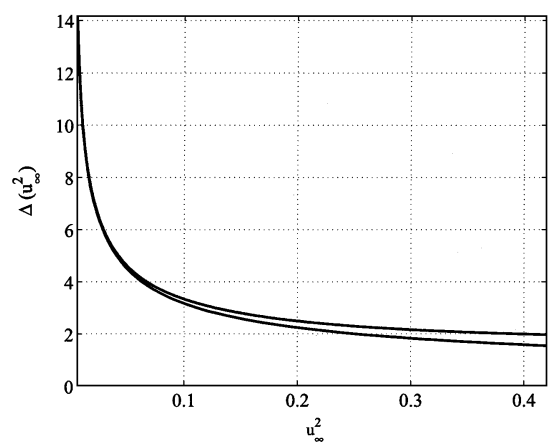

Fig. 2. Normalized soliton half-width $\Delta$ as a function of $u_{\infty}^{2}$ in the paraxial (lower curve) and nonparaxial (upper curve) regimes.

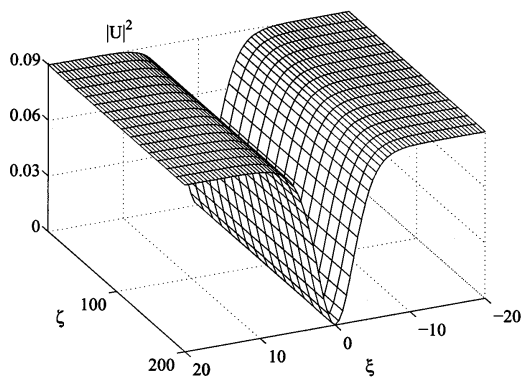

Fig. 3. Square modulus of field amplitude $|U(\xi, \zeta)|^{2}$ for four diffraction lengths obtained by solution of Eq. (2) $(\gamma=$ $-1)$ with boundary condition $U(\xi, 0)=u(\xi)$ for $u_{\infty}=0.3$.

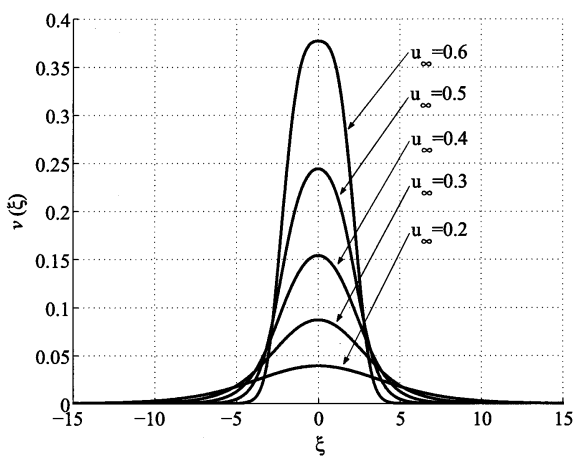

Fig. 4. Envelope $v(\xi)$ of the longitudinal field component of the soliton for the same values of $u_{\infty}$ as in Fig. 1 .

It is also possible by means of the general scheme developed in Ref. 13 to evaluate the longitudinal com- ponent $E_{z}(x, z)=i \sqrt{n_{0} /\left|n_{2}\right|} \exp (i \beta \zeta) v(\xi)$ of the soliton field. More precisely, one has ${ }^{13}$

$$
v=\left[1-\frac{4}{3}\left(u^{2}+\frac{3}{2} v^{2}\right)\right] \frac{\partial u}{\partial \xi}-\frac{4}{3} v u^{2},
$$

expressing in implicit form the longitudinal component in terms of transverse component $u$. Amplitude $v(\xi)$ obtained by solution of Eq. (10) with respect to $v$ is plotted in Fig. 4 for the soliton envelopes shown in Fig. 1. It is clearly seen, as expected, that in the nonparaxial regime (roughly corresponding to $u_{\infty}^{2}>0.2$ ) the longitudinal component becomes comparable with the transverse component.

In conclusion, we have shown that linearly polarized $(1+1) D$ nonparaxial dark solitons exist in defocusing Kerr media, provided that the asymptotic normalized intensity is not larger than $3 / 7$. The analytical relation shown in Fig. 2 between soliton width $\Delta$ and $u_{\infty}^{2}$ shows that, for $u_{\infty}^{2}>0.2$, the width is practically independent of $u_{\infty}^{2}$ and assumes the same value $\sqrt{6 / 23}$ (corresponding, in dimensional units, to a full width of $\sim \lambda / \pi$ ) that has been found for bright solitons. ${ }^{3}$ This saturation effect appears to be of a purely nonparaxial nature and constitutes the signature of nonparaxial solitons.

This research has been funded by the Istituto Nazionale di Fisica della Materia through the "Solitons Embedded in Holograms" and the Fondo per Gli Investimenti della Ricerca di Base "Space-Time Nonlinear Effects" projects and by the U.S. Air Force Office of Scientific Research (H. Schlossberg). A. Ciattoni's e-mail address is alessandro.ciattoni@aquila.infn.it.

\section{References}

1. M. Segev and G. I. Stegeman, Phys. Today 51(8), 42 (1999).

2. S. Trillo and W. Torruelas, eds. Spatial Solitons (Springer-Verlag, Berlin, 2001).

3. B. Crosignani, A. Yariv, and S. Mookherjea, Opt. Lett. 29, 1254 (2004).

4. R. de la Fuente, R. Varela, and H. Michinel, Opt. Commun. 173, 403 (2000).

5. A. Ciattoni, C. Conti, E. DelRe, P. Di Porto, B. Crosignani, and A. Yariv, Opt. Lett. 27, 734 (2002).

6. S. Chi and Q. Guo, Opt. Lett. 20, 1598 (1995).

7. B. Crosignani, P. Di Porto, and A. Yariv, Opt. Lett. 22, 778 (1997).

8. S. Blair, Chaos 10, 570 (2000).

9. G. Fibich and B. Ilan, Physica D 157, 112 (2001).

10. K. Marinov, D. I. Pushkarov, and A. Shivarova, in Soliton-Driven Photonics, A. D. Boardman and A. P. Sukhorukov, eds. (Kluwer Academic, Dordrecht, The Netherlands, 2001), pp. 293-316.

11. M. Matuszewski, W. Wasilewski, M. Trippenbach, and Y. B. Band, Opt. Commun. 221, 337 (2003).

12. G. A. Swartzlander, Jr., D. R. Andersen, J. J. Regan, H. Yin, and A. K. Kaplan, Phys. Rev. Lett. 66, 1583 (1991).

13. A. Ciattoni, P. Di Porto, B. Crosignani, and A. Yariv, J. Opt. Soc. Am. B 17, 809 (2000). 